\title{
Tempo and mode in quasispecies evolution
}

\author{
Joachim $\operatorname{Krug}^{1,2,3,4}$ \\ 1. Fachbereich Physik, Universität Essen, D-45117 Essen, Germany* \\ 2. CAMP and Department of Physics, DTU, DK-2800 Kongens Lyngby, Denmark \\ 3. Niels Bohr Institute, Blegdamsvej 17, DK-2100 Copenhagen Ø, Denmark \\ 4. Institute for Theoretical Physics, UCSB, Santa Barbara, CA 93106-4030, USA
}

November 15, 2018

\begin{abstract}
Evolutionary dynamics in an uncorrelated rugged fitness landscape is studied in the framework of Eigen's molecular quasispecies model. We consider the case of strong selection, which is analogous to the zero temperature limit in the equivalent problem of directed polymers in random media. In this limit the population is always localized at a single temporary master sequence $\sigma^{*}(t)$, and we study the statistical properties of the evolutionary trajectory which $\sigma^{*}(t)$ traces out in sequence space. Numerical results for binary sequences of length $N=10$ and exponential and uniform fitness distributions are presented. Evolution proceeds by intermittent jumps between local fitness maxima, where high lying maxima are visited more frequently by the trajectories. The probability distribution for the total time $T$ required to reach the global maximum shows a $T^{-2}$-tail, which is argued to be universal and to derive from near-degenerate fitness maxima. The total number of jumps along any given trajectory is always small, much smaller than predicted by the statistics of records for random longranged evolutionary jumps.
\end{abstract}

\footnotetext{
${ }^{*}$ Permanent address.
} 
"The concept of quasispecies is not just a model that involves any odd assumption; it shows how to view the darwinian world of replicating and mutating species from a physical viewpoint."

M. Eigen [1]

\section{Introduction and motivation}

Eigen's quasispecies theory of molecular evolution is the simplest mathematical model that incorporates the central Darwinian paradigm of natural selection acting on variability created by random mutations. The model was originally developed to understand the conditions for the maintenance of information in systems of self-instructive replicating macromolecules [2]. Such systems can be realized in the laboratory in the form of populations of RNA strands which replicate in vitro in the presence of RNA replicase, displaying a wide range of evolutionary phenomena [3, 4, 5, 6]. The notion of a quasispecies [7] refers to the structure of self-replicating populations, which typically consist of a distribution of related mutants centered around a most abundant master genotype (see below). The quasispecies structure plays an important role in the evolution of RNA viruses, where the presence of a wide range of mutants allows the virus to adapt rapidly to environmental changes 8, 9]. On the other hand, the existence of an error threshold beyond which no localized quasispecies can be maintained (see Eq.(4)) places an upper bound on the genome length of RNA viruses [10].

The mathematical structure of the quasispecies model has made it a favored entrance way for statistical physicists into the field of biological evolution日. It was first observed by Leuthäusser [14] that the discrete time dynamics (3) can be interpreted as a transfer matrix of a two-dimensional Ising model, where the genotype sequences become one-dimensional spin configurations that are coupled in the time direction through the mutation matrix (11) [15. A similar relation can be established between (3) and the transfer matrix of a polymer directed along the time axis [16, 17]. In addition, Baake and coworkers have recently exploited the equivalence between quantum spin chains and a class of kinetic evolution equations closely related to the quasispecies model, in which mutation and selection occur in parallel 12, 18.

In its most basic version, the quasispecies model is formulated in terms

\footnotetext{
${ }^{1}$ Recent articles which review this connection are [11, 12, 13].
} 
of standard chemical reaction equations written for the concentrations $n_{\sigma}(t)$ of sequences $\sigma=\left(\sigma_{1}, \ldots, \sigma_{N}\right)$, each of which is composed of $N$ symbols drawn from an alphabet of $K$ letters; the usual choice is a binary alphabet $(K=2)$, so that $\sigma_{i}$ takes the values 0 and 1 . The resulting sequence space consists then of $S=2^{N}$ points arranged on the vertices of an $N$-dimensional hypercube. Each sequence $\sigma$ reproduces at a rate $W(\sigma)$, which may be taken as a measure of its fitness [11. In the reproduction process errors occur with a mutation probability $\mu$ per site. The probability of creating a sequence $\sigma^{\prime}$ when attempting to copy sequence $\sigma$ is therefore equal to

$$
Q_{\mu}\left(\sigma \rightarrow \sigma^{\prime}\right)=\mu^{d_{H}\left(\sigma, \sigma^{\prime}\right)}(1-\mu)^{N-d_{H}\left(\sigma, \sigma^{\prime}\right)}
$$

where

$$
d_{H}\left(\sigma, \sigma^{\prime}\right)=\sum_{i=1}^{N}\left(\sigma_{i}-\sigma_{i}^{\prime}\right)^{2}
$$

is the Hamming distance between the two sequences, i.e. the number of digits in which the two differ. The dynamical evolution in discrete time is then given by

$$
n_{\sigma}(t+1)=\sum_{\sigma^{\prime}} W\left(\sigma^{\prime}\right) Q_{\mu}\left(\sigma^{\prime} \rightarrow \sigma\right) n_{\sigma^{\prime}}(t) .
$$

Introducing the constraint of a fixed number of molecules leads to nonlinear loss terms on the right hand side of (3) [7]. However since these can generally be transformed away, we ignore this complication here, at the expense of dealing with exponentially growing population numbers.

The linear form of the evolution equation (3) makes it plain that, for long times, the concentrations will approach that eigenvector of the evolution matrix $W(\sigma) Q_{\mu}\left(\sigma \rightarrow \sigma^{\prime}\right)$ which corresponds to the largest eigenvalue. Provided this eigenvector is localized in sequence space, it defines the quasispecies: A distribution of related mutants centered around the master sequence, which usually is the sequence with the maximum replication rate $W(\sigma)$. The most celebrated property of the model is its prediction of a sharp error threshold, where the quasispecies delocalizes, and the population spreads uniformly over sequence space. In terms of the sequence length $N$ and the mutation probability $\mu$, the condition for a localized quasispecies takes the form [2, 7]

$$
N<N_{\max }=\frac{\ln A}{\mu},
$$

\footnotetext{
${ }^{2}$ Similar equations arise in classical population genetics [12].
} 
where $A$ denotes the selective advantage, a measure for the superiority of the master sequence compared to the other sequences. For the simplest case of a single peak fitness landscape, where the master sequence replicates at rate $W_{0}$ and all other sequences replicate at rate $W_{1}<W_{0}$, the selective advantage is $A=W_{0} / W_{1}$, while for randomly distributed replication rates it is a functional of the rate distribution [19, 20]. In terms of the physical analogies described above, the error threshold phenomenon is equivalent to the thermal phase transition in the Ising model [14, 15, 18, 20, 21] and to the thermal unbinding of a directed polymer bound to an attractive columnar defect along the time direction [16, 17].

Much less appears to be known about the evolutionary dynamics of the model, that is, the approach to the final quasispecies distribution from an initial localized or delocalized state. It was first pointed out by McCaskill [19, 22 that this dynamics should take the form of a "slowing optimization walk" through a succession of metastable states which correspond, in some sense, to local maxima in the fitness landscape. The separation of time scales between the (long) residence time in a metastable state and the (brief) transition time to the next maximum implies a punctuated pattern of evolution [23, 24, 25], which can be analyzed in analogy to variable range hopping in condensed matter physics [26, 27, 28].

We should concede from the outset that the deterministic rate equations (3) are not an entirely appropriate description for this evolutionary regime, since the transition between two local maxima proceeds through the tails of the localized, metastable quasispecies, where the population numbers are small and fluctuations due to the finite number of molecules cannot be ignored [22, 28, 29]. It seemed nevertheless worthwhile to explore these questions within the most basic, deterministic model, before turning to more sophisticated approaches.

The present paper reports on some preliminary results from such an investigation. To avoid the complications due to a finite error threshold, we consider a strong selection limit (to be described in the next section), in which the population is localized at a single site in sequence space at all times. This allows a direct comparison with simple schematic models of evolutionary dynamics, such as adaptive walks [30, 31, 32, 33] and record dynamics 34, 35, 36]. Adaptive walks describe the evolution of a genetically homogeneous population under the assumption that deleterious mutations (which decrease the fitness) are eliminated, while advantageous mutations spread instantaneously. The population then performs an uphill walk in 
the fitness landscape, which terminates at a local maximum where no fitter one-step neighbors are available. In contrast, in the quasispecies model the population evolves through a chain of local fitness maxima which progresses all the way to the global optimum. Some qualitative properties of these evolutionary trajectories are described in Section o 0 , while Section 1 focuses on a specific statistical feature, the total evolution time. A comparison with record dynamics is provided in Section 5, and some open questions are formulated in Section 6 .

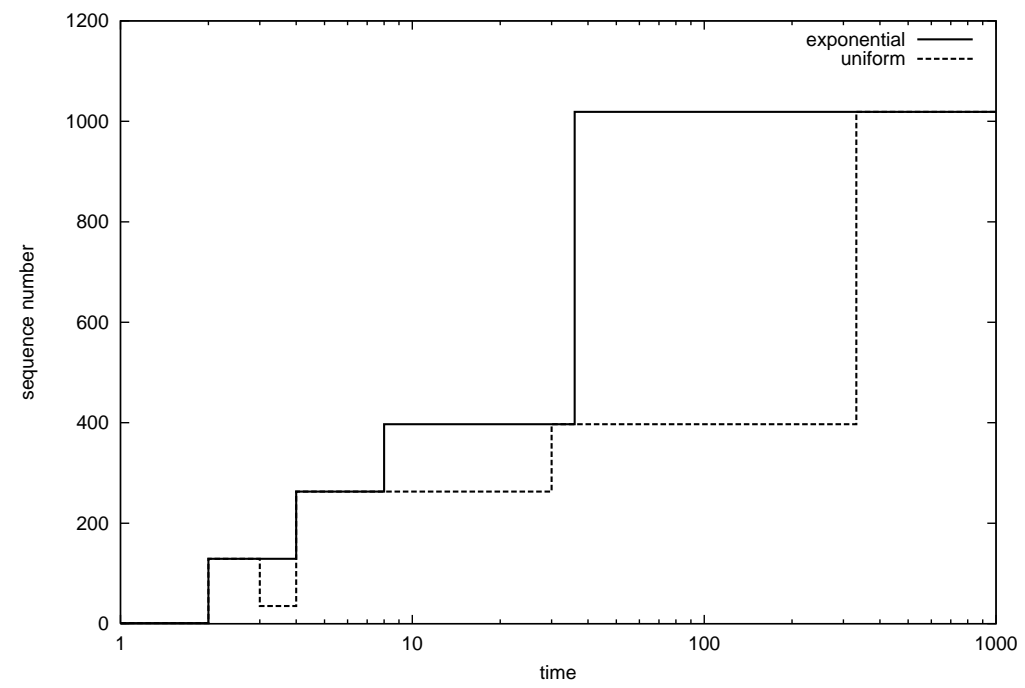

Figure 1: Two evolutionary trajectories generated in two fitness landscapes with identical ordering of fitnesses but different fitness distributions. The monotonic increase in sequence number for the exponential distribution is fortuitous - there is no correspondence between the position of a sequence on the $y$-axis and its fitness

Throughout the paper we will consider maximally rugged fitness landscapes, in which the reproduction rate $W(\sigma)$ is chosen independently and randomly for each sequence. In contrast to simpler permutation invariant landscapes [12], this makes it necessary to store all $2^{N}$ sequences during the iteration of the evolution equations ${ }^{3}$, restricting our numerical treatment to

\footnotetext{
${ }^{3} \mathrm{An}$ approximate scheme which reduces the storage requirement from $2^{N}$ to $N$ is described in 38.
} 
rather short sequences; the results shown here are for $N=10$. A systematic analysis of the dependence on sequence length will be presented elsewhere 38.

\section{The strong selection limit}

The form of the strong selection limit is motivated by the analogy with the zero temperature limit in the associated problems of statistical physics. Following Peliti [11, 21] we introduce an inverse "selective temperature" $k>0$ by writing the reproduction rates in the form

$$
W(\sigma)=e^{k F(\sigma)} .
$$

We want to take the strong selection limit $k \rightarrow \infty$ in such a way that only a few mutations occur in each time step. This requires to scale the mutation rate as

$$
\mu=e^{-k \gamma}
$$

where $\gamma>0$ is a constant. Inserting (5) and (6) into (3) it is clear that the sequence concentrations will grow for large $k$ as

$$
n_{\sigma}(t)=e^{k E(\sigma, t)} .
$$

In the limit $k \rightarrow \infty$ the evolution equation (3) then reduces to the recursion

$$
E(\sigma, t+1)=\max _{\sigma^{\prime}}\left[E\left(\sigma^{\prime}, t\right)+F\left(\sigma^{\prime}\right)-\gamma d_{H}\left(\sigma, \sigma^{\prime}\right)\right] .
$$

Since the term $-\gamma d_{H}$ suppresses mutations to far away sequences, we expect similar behavior for a model in which only nearest neighbor mutations are allowed,

$$
E(\sigma, t+1)=\max _{d_{H}\left(\sigma, \sigma^{\prime}\right) \leq 1}\left[E\left(\sigma^{\prime}, t\right)+F\left(\sigma^{\prime}\right)-\gamma d_{H}\left(\sigma, \sigma^{\prime}\right)\right] .
$$

All results shown in this paper were obtained using the nearest neighbor rule (9), with the parameter $\gamma$ set to unity

We still need to specify the probability distribution $p(F)$ of the fitnesses $F(\sigma)$. Two choices will be considered: The exponential distribution $p(F)=$ $e^{-F}, F \geq 0$, and a uniform distribution on the interval $[0, S]$, where $S=2^{N}$. The reason for this particular scaling of the width of the uniform distribution will become clear below in Section 4 .

\footnotetext{
${ }^{4}$ For a discussion of the differences between the rules (8) and (9), see [38].
} 


\section{$3 \quad$ Evolutionary trajectories}

It is evident from (7) that, in the strong selection limit $k \rightarrow \infty$, the entire population resides at the global maximum of the function $E(\sigma, t)$. The position of this maximum in sequence space will be referred to as the master sequence at time $t$, and denoted by $\sigma^{*}(t)$. At time $t=0$ the master sequence is placed at a randomly chosen point $\sigma^{(i)}$ by setting $E\left(\sigma^{(i)}, t\right)=0$ and $E(\sigma, t)=-\infty$ for $\sigma \neq \sigma^{(i)}$. The subsequent time evolution $\sigma^{*}(t)$ defines an evolutionary trajectory.

Inspection shows that, after one or two time steps, such a trajectory passes exclusively through local fitness maxima, and eventually, after a total evolution time $T$, it invariably reaches the global fitness maximum. During the evolution, the master sequence spends increasingly long time intervals at local maxima of increasing fitness, with a few abrupt transitions in between (Figure 11). The number of transitions is small (see Figure 5), much smaller than the number of local fitness maxima, which is on average equal to $2^{N} /(N+1) \approx 93$ [30. This implies that most local maxima are bypassed by a typical trajectory.

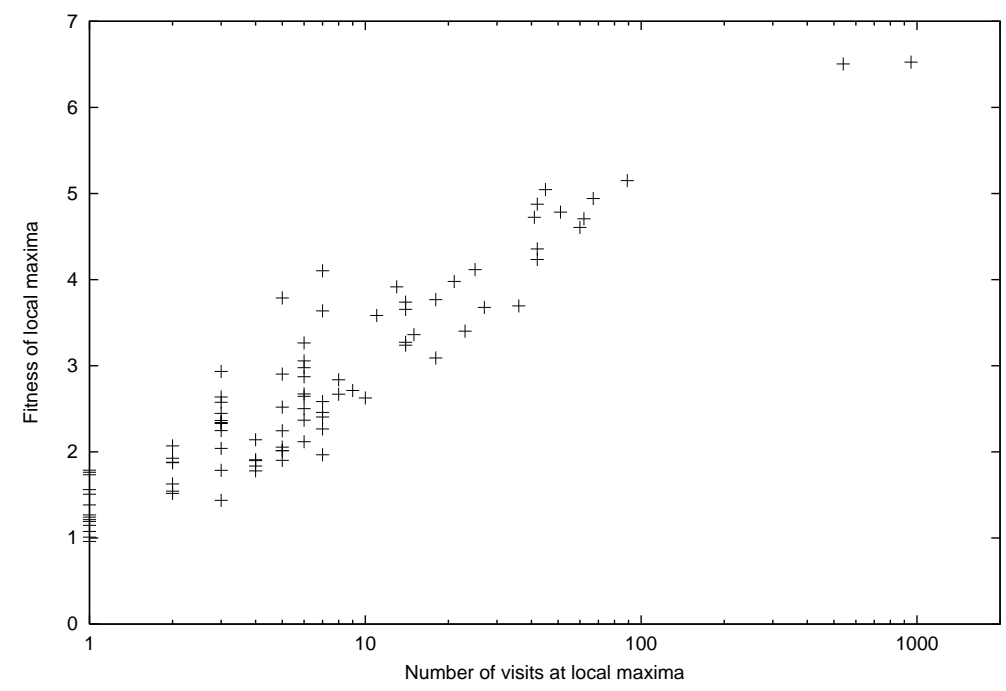

Figure 2: Fitness $F(\sigma)$ vs. the number of visits for all local maxima in a fitness landscape with exponential fitness distribution $p(F)$. The particular landscape used here is near degenerate (gap size $\epsilon \approx 0.02$ ) 
A quantification of this statement is shown in Figure 2, in which the fitness $F$ of local maxima is plotted against the number of times it is visited by an evolutionary trajectory. These data were generated by going through all possible starting points $\sigma^{(i)}$ in a fixed fitness landscape. The figure shows a roughly linear correlation between the fitness of a maximum and the logarithm of the number of visits.

In relation to adaptive walks [30, 31, 32, 33, which respond only to the relative ordering of fitnesses and not to their actual values, it is of interest to ask to what extent the set of maxima visited by a given trajectory is determined by the ordering of fitnesses. For this reason Figure 11 shows two trajectories evolving in landscapes which were generated using the same random numbers - thus having identical ordering of fitnesses - but with different fitness distributions. It can be seen that the set of local maxima visited by the two trajectories is almost identical, apart from a small detour taken by the "uniform" trajectory, but the timing of the evolutionary transitions is markedly different in the two cases. With reference to G.G. Simpson's classic treatise [23], we may say that the fitness distribution affects only the tempo, but not the mode of quasispecies evolution. For a quantitative analysis of the temporal aspects we next turn to the distribution of evolution times.

\section{Distribution of evolution times}

Figure 3 shows the distribution $P(T)$ of the number of time steps $T$ required to reach the global fitness maximum, obtained by averaging over 500000 landscapes with exponential and uniform fitness distributions. The time distribution for the exponential case displays a distinct maximum around $T=7$, followed by a slowly decaying tail which is well described by the power law

$$
P(T) \sim T^{-2}
$$

over roughly two decades. The distribution for the uniform case is much broader, but a similar power law tail can be seen for times $T \geq 500$.

The power law (10) appears to be a simple consequence of the order statistics of uncorrelated fitness landscapes. Let $F^{(1)}>F^{(2)}>\ldots>F^{(S)}$ be a realization of fitnesses arranged in decreasing order. As a measure of the spread in fitnesses among the most fit sequences we introduce the fitness gap

$$
\epsilon=F^{(1)}-F^{(2)}>0
$$




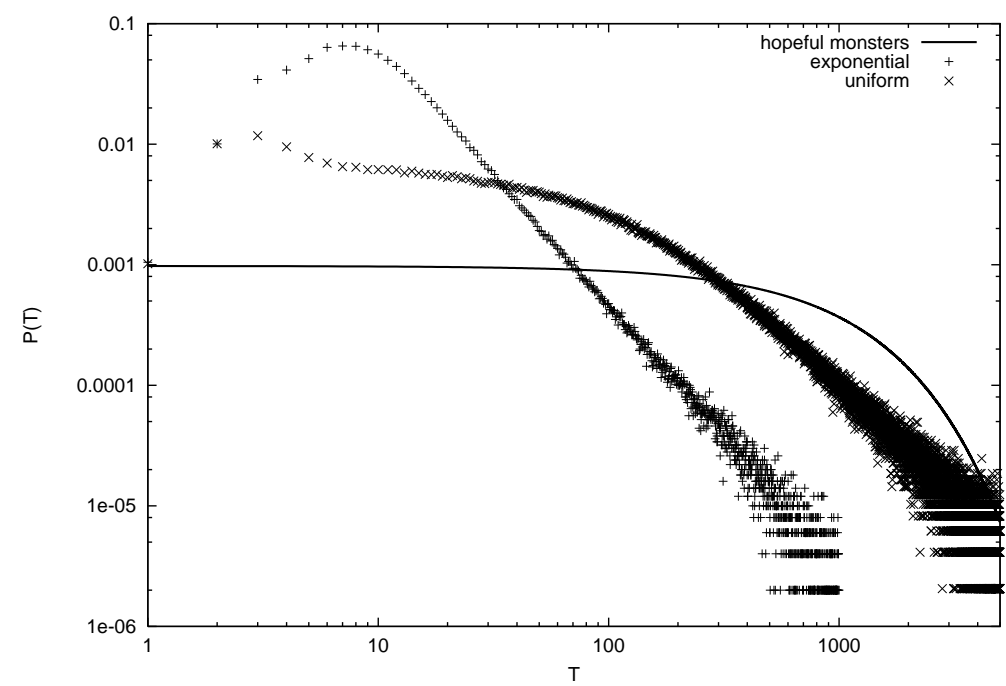

Figure 3: Distribution of the total time $T$ required to reach the global fitness maximum. Symbols show data obtained by averaging over 500000 uncorrelated fitness landscapes with exponential $(+)$ and uniform $(\times)$ fitness distributions, while the full line shows the distribution (17) obtained for record dynamics. The simulations with exponential fitness distribution were stopped after 1000 time steps

which is a random variable characteristic of each fitness landscape. In the late stage of evolution the population will typically make a a transition from the second best sequence $\sigma^{(2)}$ (or some local fitness maximum with comparable fitness 可) to the globally optimal sequence $\sigma^{(1)}$. From the evolution rule (9) it is easy to see that, for small $\epsilon$, this transition will require a time of the order of

$$
T \approx a(N) / \epsilon
$$

where the coefficient $a$ is determined by the early stages of the evolution process [38. Thus given the gap distribution $P_{\mathrm{g}}(\epsilon)$ the tail of the distribution of evolution times can be estimated to be

$$
P(T) \approx a T^{-2} P_{\mathrm{g}}(a / T),
$$

and a $T^{-2}$ power law follows for $T \gg a$, provided that $0<P_{\mathrm{g}}(0)<\infty$. The gap distribution is given by $P_{\mathrm{g}}(\epsilon)=e^{-\epsilon}$ both for exponentially distributed

\footnotetext{
${ }^{5}$ In fact $\sigma^{(2)}$ is a local fitness maximum with high probability $1-N /\left(2^{N}-1\right) \approx 0.990$.
} 
fitnesses, and for fitnesses distributed uniformly between 0 and $S$, when $S$ is large [39]. The striking difference between the two evolution time distributions seen in Figure 3 is related to the different scaling of the coefficient $a(N)$ in (12) with sequence length: In the exponential case $a(N) \sim N^{3 / 2}$, while in the uniform case $a(N) \sim \sqrt{N} S$ [38].

To compute $P_{\mathrm{g}}(0)$ for general fitness distributions, note first that the joint distribution of $F^{(1)}$ and $F^{(2)}$ is given by 40,

$$
P_{2}\left(F^{(1)}, F^{(2)}\right)=S(S-1) p_{c}\left(F^{(2)}\right)^{S-2} p\left(F^{(1)}\right) p\left(F^{(2)}\right)
$$

where $p_{c}(F)=\int_{0}^{F} d F^{\prime} p\left(F^{\prime}\right)$ denotes the cumulative fitness distribution. The cumulative gap distribution is obtained by integration,

$$
\begin{gathered}
\operatorname{Prob}\left[F^{(1)}-F^{(2)}<\epsilon\right]= \\
S(S-1) \int_{0}^{\infty} d F^{(2)} p\left(F^{(2)}\right) p_{c}\left(F^{(2)}\right)^{S-2} \int_{F^{(2)}}^{F^{(2)}+\epsilon} d F^{(1)} p\left(F^{(1)}\right),
\end{gathered}
$$

which tends to $P_{\mathrm{g}}(0) \epsilon$ for $\epsilon \rightarrow 0$. Thus we conclude that

$$
P_{g}(0)=S(S-1) \int_{0}^{\infty} d F p(F)^{2} p_{c}(F)^{S-2}
$$

which is clearly finite and nonzero.

The relationship (13) implies that the near-degenerate fitness landscapes, which have very small gaps, are the ones that give rise to anomalously long evolution times. Figure 1 illustrates this connection. The data shown as crosses were obtained from an average over exponential fitness landscapes, for which the fitness gap $\epsilon$ was increased artificially by increasing the global fitness maximum according to $F^{(1)} \rightarrow F^{(1)}+1$. This is seen to immediately remove the power law tail $(\overline{10})$.

\section{Comparison to record dynamics}

A simple schematic analogue of the nonstationary (ever slowing) evolution process found in the quasispecies model is provided by the dynamics of records 34, 35, 36, 39, which is equivalent to evolution by long-ranged random mutations known in the classical literature as the theory of "hopeful monsters" [23, 30]. In the present context it reduces to the following rule for the motion of the master sequence $\sigma^{*}(t)$ in sequence space: At each time 


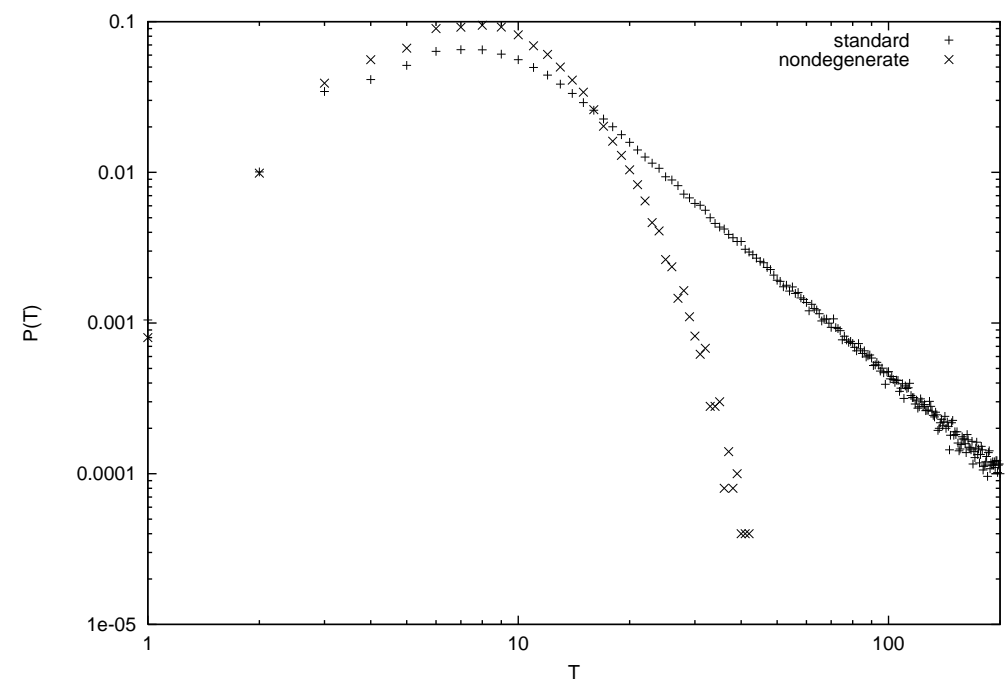

Figure 4: Comparison of the distribution of evolution times for exponentially distributed fitnesses $(+)$ with an ensemble of landscapes for which neardegeneracies (small gaps) have been removed $(x)$. The latter data were averaged over 50000 realizations

step, the population attempts a jump to another, randomly chosen sequence $\sigma^{\prime} \neq \sigma^{*}(t)$. The move is accepted, and $\sigma^{*}(t+1)=\sigma^{\prime}$, if $F\left(\sigma^{\prime}\right)>F\left(\sigma^{*}(t)\right)$; otherwise it is discarded and $\sigma^{*}(t+1)=\sigma^{*}(t)$. Thus the current sequence $\sigma^{*}(t)$ represents the fitness record among the sequences which the population has encountered so far.

Clearly this process gives rise to a step-like, punctuated pattern which is qualitatively similar to that shown above in Figure 11. Here we are concerned with a quantitative comparison of statistical properties. Let us first compute the probability distribution of the total evolution time $T$ for the record dynamics. Since the probability of finding the global fitness maximum in any jump is $1 / S$, the probability that it has not been found up to time $t$ is $(1-1 / S)^{t} \approx e^{-t / S}$ for large $S$ and $t$. Taking the derivative one obtains

$$
P(T)=S^{-1} e^{-T / S}
$$

The typical evolution times are of the order of the number of sequences, much larger than in the quasipecies model. This demonstrates impressively the "guided" nature of quasispecies evolution [7], which is much more efficient 


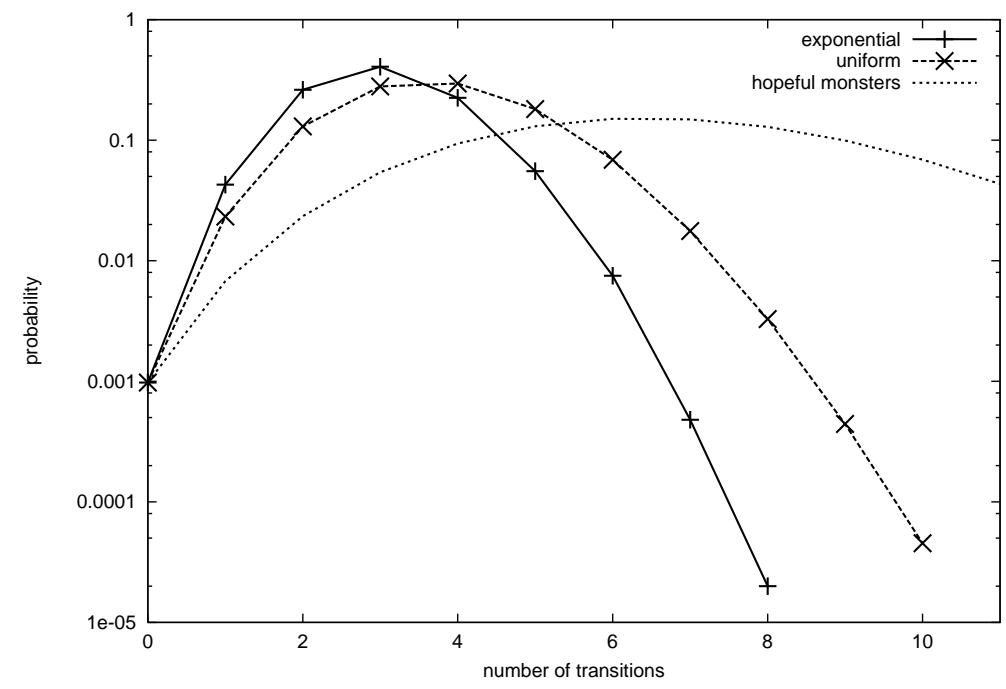

Figure 5: Probability distributions for the total number of evolutionary jumps required to reach the global fitness maximum. Symbols show data obtained by averaging over 500000 fitness landscapes with exponential (+) and uniform $(x)$ fitness distributions, while the dotted line is the log-Poisson distribution (18) predicted by record dynamics

than a random search. Figure 3 shows how broad the distribution (17) is compared to that of the quasispecies model. Note, however, that for very long times (longer than $S$ ) Eq.(17) decays exponentially, faster than the degeneracy-induced power law (10). Taken literally, Eq.(10) implies that the mean evolution time is infinite.

Next we consider the distribution $P_{n}$ of the total number $n$ of evolutionary jumps which occur on the way to the global fitness maximum. Adapting the results of Sibani and collaborators [36, 37], for the case of record dynamics we find that $P_{n}$ is a Poisson distribution with parameter $\ln S$,

$$
P_{n} \approx S^{-1} \frac{(\ln S)^{n-1}}{(n-1) !} .
$$

In Figure 5 this is compared to numerical data obtained for the quasispecies model. Again the distributions for the quasispecies dynamics are much narrower, showing that less transitions are required to reach the global maximum. Simulations for longer sequences show that the mean number of 
transitions increases sublinearly in $N$, more slowly than the linear behavior predicted by (18) [38, 41].

\section{Outlook}

The simplicity of the strong selection dynamics (8,9) suggests to use it for a dynamical characterization of different kinds of fitness landscapes. In contrast to the random landscapes considered here, realistic fitness landscapes obtained e.g. from RNA folding contain extended neutral networks in sequence space, in which the fitness (defined in terms of the RNA secondary structure) does not change [42]. Central concepts of quasispecies theory have been extended to such landscapes [43]. Extended neutrality provides a distinct mechanism for the appearance of punctuation patterns in evolution, since changes in the genotype do not show up in the phenotype, as long as the former moves within a neutral network [44].

Another interesting direction for further research inspired by the analogy with directed polymers is to include effects of environmental fluctuations, which amounts to making the fitness landscape time-dependent [45]. In the directed polymer analogy, the issue is the interplay between columnar disorder, which is provided by the time-independent part of the landscape, and point disorder modeling the time-dependent variations 46]. It is well known that point disorder can depin a polymer from an attractive columnar defect in much the same way as thermal fluctuations [47. This suggests the intriguing possibility of an error threshold delocalization phenomenon induced by environmental fluctuations.

Acknowledgements. I would like to thank T. Halpin-Healy and C. Karl for their contributions to this project, and K. Sneppen, H. Flyvbjerg and L. Peliti for useful discussions. This work has been supported in part by NATO within CRG.960662, and by NSF under Grant No. PHY99-07949.

\section{References}

[1] M. Eigen: Trends in Microbiology 4, 216 (1996)

[2] M. Eigen: Naturwissenschaften 58, 465 (1971) 
[3] D.R. Mills, R.L. Peterson, S. Spiegelman: Proc. Natl. Acad. Sci. USA 58, 217 (1967).

[4] C.K. Biebricher: 'Darwinian Selection of Self-Replicating RNA Molecules'. In: Evolutionary Biology, Vol. 16, ed. by M.K. Hecht, B. Wallace, G.T. Prance (Plenum, New York 1983) pp. 1-52

[5] C.K. Biebricher, W.C. Gardiner: Biophys. Chem. 66, 179 (1997)

[6] J.S. McCaskill, G.J. Bauer: Proc. Natl. Acad. Sci. USA 90, 4191 (1993)

[7] M. Eigen, J. McCaskill, P. Schuster: Adv. Chem. Phys. 75, 149 (1989)

[8] E. Domingo: Clinical and Diagnostic Virology 10, 97 (1998)

[9] R.V. Solé, R. Ferrer, I. González-García, J. Quer, E. Domingo: J. theor. Biol. 198, 47 (1999)

[10] M. Eigen and C.K. Biebricher: 'Role of Genome Variation in Virus Evolution'. In: RNA Genetics. Vol. III: Variability of Virus Genomes, ed. by E. Domingo, J.J. Holland, P. Ahlquist (CRC Press, Boca Raton, FL, 1988) pp. 211-245

[11] L. Peliti: 'Introduction to the statistical theory of Darwinian evolution'. cond-mat/9712027

[12] E. Baake, W. Gabriel: 'Biological evolution through mutation, selection, and drift: An introductory review'. In: Annual Reviews of Computational Physics VII, ed. by D. Stauffer (World Scientific, Singapore, 2000) pp. 203-264

[13] B. Drossel: 'Biological Evolution and Statistical Physics'. cond-mat/0101409 (to appear in Adv. Phys.)

[14] I. Leuthäusser: J. Stat. Phys. 48, 343 (1987)

[15] P. Tarazona: Phys. Rev. A 45, 6038 (1992)

[16] S. Galluccio, R. Graber, Y.-C. Zhang: J. Phys. A 29, L249 (1996)

[17] S. Galluccio: Phys. Rev. E 56, 4526 (1997)

[18] E. Baake, M. Baake, H. Wagner: Phys. Rev. Lett. 78, 559 (1997) 
[19] J.S. McCaskill: J. Chem. Phys. 80, 5194 (1984)

[20] S. Franz, L. Peliti, M. Sellitto: J. Phys. A 26, L1195 (1993)

[21] S. Franz, L. Peliti: J. Phys. A 30, 4481 (1997)

[22] J.S. McCaskill: Biol. Cybern. 50, 63 (1984)

[23] G.G. Simpson: Tempo and Mode in Evolution (Columbia University Press, New York, 1944)

[24] C.M. Newman, J.E. Cohen, C. Kipnis: Nature 315, 400 (1985)

[25] S.J. Gould, N. Eldredge: Nature 366, 223 (1993)

[26] W. Ebeling, A. Engel, B. Esser, R. Feistel: J. Stat. Phys. 37, 369 (1984)

[27] J. Krug, T. Halpin-Healy: J. Phys. I France 3, 2179 (1993)

[28] Y.C. Zhang: Phys. Rev. E 55, R3817 (1997)

[29] D. Alves, J.F. Fontanari: Phys. Rev. E 57, 7008 (1998)

[30] S. Kauffman, S. Levin: J. theor. Biol. 128, 11 (1987)

[31] C.A. Macken, A.S. Perelson: Proc. Natl. Acad. Sci. USA 86, 6191 (1989)

[32] C.A. Macken, P.S. Hagan, A.S. Perelson: SIAM J. Appl. Math. 51, 799 $(1991)$

[33] H. Flyvbjerg, B. Lautrup: Phys. Rev. A 46, 6714 (1992)

[34] P. Sibani, M.R. Schmidt, P. Alstrøm: Phys. Rev. Lett. 75, 2055 (1995)

[35] P. Sibani: Phys. Rev. Lett. 79, 1413 (1997)

[36] P. Sibani, M. Brandt, P. Alstrøm: Int. J. Mod. Phys. B 12, 361 (1998)

[37] P. Sibani, P.B. Littlewood: Phys. Rev. Lett. 71, 1482 (1993).

[38] J. Krug and C. Karl (in preparation)

[39] W. Feller, Introduction to Probability Theory and Its Applications, Vol. 2 (Wiley, New York 1971) 
[40] H.A. David, Order Statistics (Wiley, New York 1970)

[41] C. Karl: Diploma thesis (University of Essen, 2001)

[42] P. Schuster: Biophys. Chem. 66, 75 (1997)

[43] C. Reidys, C.V. Forst, P. Schuster: Bull. Math. Biol. 63, 57 (2001)

[44] P. Schuster, W. Fontana: Physica D 133, 427 (1999)

[45] M. Nilsson, N. Snoad: Phys. Rev. Lett. 84, 191 (2000)

[46] I. Arsenin, T. Halpin-Healy, J. Krug: Phys. Rev. E 49, R3561 (1994)

[47] L. Balents, M. Kardar: Phys. Rev. B 49, 13030 (1994) 